\title{
Applications of Chitin and Chitosan in Industry and Medical Science: A Review
}

\author{
Shanta Pokhrel ${ }^{1}$, Paras Nath Yadav $^{\mathbf{1}}$ and Rameshwar Adhikari ${ }^{\mathbf{1}, 2}$ \\ ${ }^{1}$ Central Department of Chemistry, Tribhuvan University, Kathmandu, Nepal \\ ${ }^{2}$ Research Centre for Applied Science and Technology (RECAST), Tribhuvan University, \\ Kathmandu, Nepal \\ e-mail: shantabhattarai2014@gmail.com
}

\begin{abstract}
Chitin can be extracted from the exoskeletons of crustaceans, insects and mollusks and the cell wall of microorganisms. It can be converted into chitosan by deacetylation process. Chitosan shows more versatility than chitin due to its solubility and reactive free amino group $\left(-\mathrm{NH}_{2}\right)$. This article helps to understand the importance and characteristics of chitin and chitosan by their various aspects such as properties and medical and industrial applications.
\end{abstract}

Key words: biomaterial, chitin, chitosan, medical application, biopolymer

\section{Introduction}

The meaning of biopolymer is something, that is originated from living beings; made by the combination of many molecules. There are large number of natural polymers considered as biomaterials such as collagen, chitin, silk, alginate, starch and elastin (Correlo et al.2005). The problem associated with synthetic polymers such as toxicity and non biodegradability is suppressed by the use of biopolymers. The important characteristic of biopolymer is its ability to degrade by natural enzymes with their immunogenic behavior. The development of biomaterials using natural polymers is an important and promising channel for research.

Chitin is the second most abundant biopolymer found in nature. It is mainly derived from the exoskeletons of crustaceans, insects, mollusks and the cell wall of microorganisms (Muzzarelli 1977). Chitin is a copolymer of $\mathrm{N}$-acetyl-D-glucosamine units linked with â-(1-4) glycosidic bond, where N-acetyl-Dglucosamine units are predominant in the polymeric chain.

Chitosan is the deacetylated derivative of chitin, which is chemically defined as a copolymer of á- $(1,4)$ glucosamine $\left(\mathrm{C}_{6} \mathrm{H}_{11} \mathrm{O}_{4} \mathrm{~N}\right)_{\mathrm{n}}$, having different number of $\mathrm{N}$-acetyl groups (Zvezdova 2010). It is white to light red solid powder, insoluble in water but soluble in organic acids.

The structures of chitin and chitosan are presented in Scheme 1.

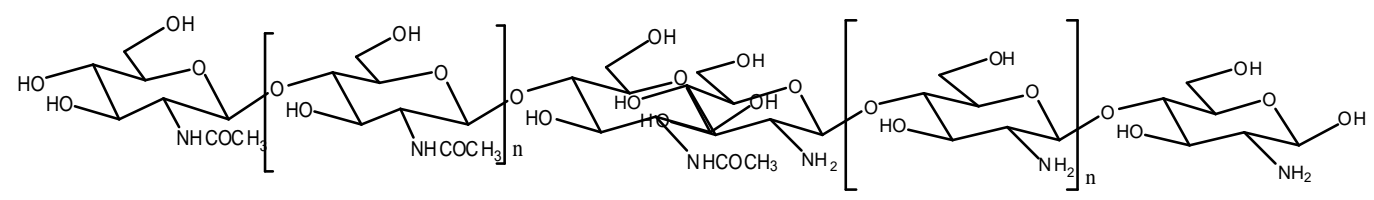

Chitin

Chitosan

Scheme1. Polymer chain of chitin and chitosan where $n$ stands for the degree of polymerization 
Chitin can be isolated from crustacean shells by chemical process, which was reported by Acosta et al. (1995) and Zvezdova (2010). A general scheme is presented in Fig. 1 and involves the following steps.

(a) Demineralization: It involves acid treatment (mainly with $\mathrm{HCl}$ ) which removes inorganic matters (mainly calcium carbonate). (b) Deproteinization: It includes the extraction of protein matter in alkaline medium (mainly with $\mathrm{NaOH})$.

(c) Decolourization: It involves bleaching of the product by chemical reagents to achieve colourless product.

The conversion of chitin into chitosan has been shown in Scheme 2.
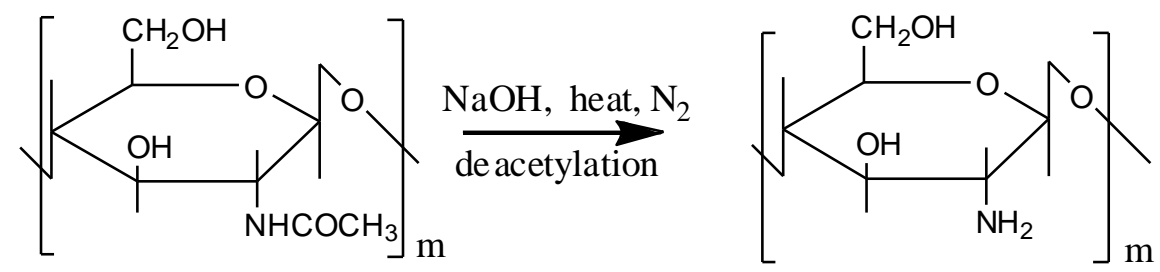

Scheme 2. Schematic representation for the conversion of chitin into chitosan

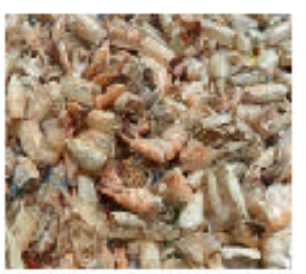

Prawn Shells

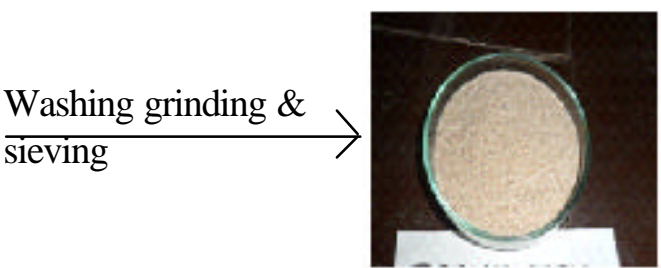

Fine powder: $\leq 500 \mu \mathrm{m}$

\section{$7 \% \mathrm{HCI} / 24 \mathrm{~h}$}

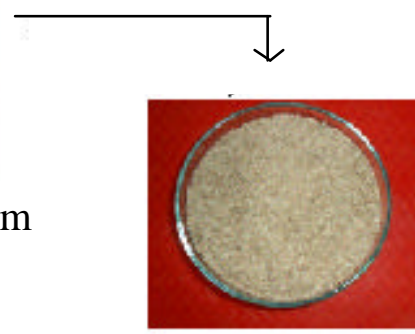

Demineralised powder

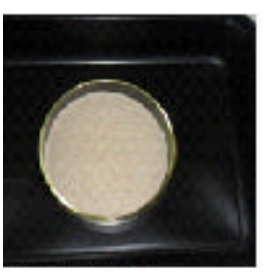

Chitosan

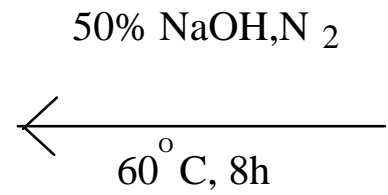

$60 \mathrm{C}, 8 \mathrm{~h}$

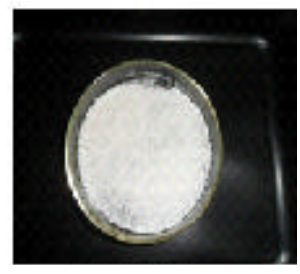

Chitin

Fig. 1. Photographs showing different steps of isolation of chitosan from prawn shells using method adapted from literature (Zvezdova 2010)

Chitin and chitosan are the naturally abundant and renewable polymers having excellent properties such as biodegradability, biocompatibility, non-toxicity and adsorption (Hudson \& Smith 1998). The reaction of chitosan is more versatile than cellulose due to the presence of $-\mathrm{NH}_{2}$ groups (Dutta et al. 2004).
Like cellulose, chitin and chitosan can undergo many reactions such as etherification, esterification and crosslinking (Hon 1996). The main parameter that affects the characteristics of chitosan is its molecular weight (Zhou et al. 2003) and degree of deacetylation (representing the proportion of deacetylated units). 
Unlike other naturally occurring polysaccharides such as cellulose, dextran, pectin, alginic acid that are either acidic or neutral, chitosan is highly basic in nature. Due to this unique property, chitosan has several functional properties such as polyoxysalt formation, ability to form films, chelate metal ion and optical structural characteristics (Majeti 2000, Hench 1998).

\section{Chemical properties of chitosan}

This biopolymer has a number of chemical properties that make it suitable for several biomedical applications. Some of them are listed below (Dutta et al. 2004).

1. Chitosan is a linear polyamine.

2. It has reactive amino groups $\left(-\mathrm{NH}_{2}\right)$.

3. There is availability of reactive hydroxyl groups $(-\mathrm{OH})$.

4. It has chelating ability for many transitional metal ions.

\section{Biological properties of chitosan}

Besides the various chemical properties, chitosan shows diverse biological properties which are summarized (Venter et al.2006, Younes \& Rinaudo 2015) as follows:

1. Biocompatible:

i. Natural polymer

ii. Safe and non-toxic

iii. Biodegradable to normal body constituents

2. Binds to mammalian and microbial cells aggressively

3. Regenerative effect on connective gum tissue

4. Accelerates the formation of osteoblast responsible for bone formation

5. Haemostatic (causes stop bleeding)

6. Fungistatic (inhibiting the growth of fungi)

7. Spermicidal (birth control)

8. Antitumor or Anticancer (inhibiting the growth of tumor or cells)

9. Anticholesteremic (cholesterol lowering agent)

10. Central nervous system depressant (slow down the brain activity)

11. Immunoadjuvant (involved in the improvement of immune response)

\section{Applications of chitin and chitosan}

The poor solubility of chitin causes limitation in its applications. Besides these limitations, its various applications have been reported such as raw material for man-made fibers as absorbable sutures and wound dressing materials besides these chitin and chitosan fibers are also used in waste water treatment (Sridhari et al. 2000)

Chitin and chitosan have potential applications in hair care. Chitin and acylated chitosan with an organic diacid anhydride are used for skin care. Chitosan can be used in shampoos, hair colorants, styling lotions, hair sprays and hair tonics (Rinaudo 2006). Chitin and chitosan are found to be used in creams as a moisturizer, nail enamel foundation, eye shadow and lipstick cleansing materials and bathing agents. Chitin and chitosan are used in toothpaste, mouthwashes and chewing gum. Chitin is also used as dental filler (Dutta et al. 2004).

Chitosan act as chelating agent and heavy metals trapper (Khor \& Lim 2003). Chitosan N-benzyl sulphonate derivatives are used as sorbents for the removal of metal ions in acidic medium and chitosan can also be used to remove the color from dye house effluents as reported by Weltroswki et al. (1996). Chitin and chitosan are also found to be used effectively to remove arsenic from contaminated drinking water as well as to remove petroleum products from waste water (Saha \& Sarkar 2013).

Chitosan is non-toxic therefore it is used in food industry. Microcrystalline chitin (MCC) is used as flavouring and colouring agent, shelf life as well as dietary fibre in baked food (Jianglian \& Shaoying 2013).

Chitin treated seeds (wheat) have shown the growth accelerating and growth enhancing effects. The addition of chitin to the soil reduces the root knot worm infestations and supression of fungal pathogens (Dutta et al. 2004).

Tissue engineering is the development and manipulation of laboratory-grown cells, tissues or organs that would replace or support the function of defective or injured parts of the body (Khor \& Lim 2003, Vankatesam \& Kim 2010). The special attention on chitosan has been paid for the repair of articular cartilage. Microporous chitosan/calcium phosphate composite scaffolds have been synthsized and characterized for tissue engineering. They reported that chitosan provides a scaffold form and calcium phosphates encourages osteoblast attachement and 
strengthens the scaffold (Zhang \& Zhang 2001, Elzein et al. 2002).

Chitosan show good effect on wound dressing/wound healing process. Chitin can also be used as a coating on normal biomedical materials. Standard silk and catgut sutures coated with regenerated chitin or chitosan show wound healing activities slightly lower than the chitin fibre (Murakami et al. 2010).

Chitin and chitosan are inexpensive and non hazardious. They can be used for the prepartion of dosage forms in commercial drugs (Andrady \& Xu 1997) such as spread dry chitosan acetate and ethyl cellulose can be used as new compression coats for 5aminosalicylic acid (ASA) tablets was reported by Nunthanid et al. (2009). Chitin/chitosan controlled delivery systems are in developing stage. It is being used for a wide variety of reagents in several environments. (Surini et al. 2003).

Gene therapy is a treatment of human disorders by introducing the genetic material into specific target cells of a patient where the production of the encoded protein occurs (Corsi et al. 2003).

The development of efficient and safe gene carrier systems that are capable of transferring DNA in the cells is a major goal of the gene therapy. Several literature survey show that chitosan is a suitable material for efficient non-viral gene therapy (Jayakumar et al. 2010).

Besides many good characteristics, chitosan has growth-inhibition effect on tumor cells (Carreno-Gomez \& Duncan 1997). The ligand-targated approach is expected to deliver drugs to tumor tissues selectively with high efficiency. Although in vivo studies of targated chitosan nanoparticles are currently limited, results from in vitro studies have demonstrated their promise for applications in cancer treatment and diagnosis (Hang 2010).

As chitosan shows antimicrobial activity so its film have shown great promise for their applicaton in food preservation. The antimicrobial activity limits or prevents microbial growth by extending the interval period and reducing the growth rate or decreasing live counts of microorganisms (Han 2000) which support the chitosan film for its potentiality in the packaging of food items and food preservation.
Gavhane et al. (2013) reported wide biomedical applications of chitin and chitosan, however, he also highlighted some limitations such as allergy and constipation. Furhermore people with intestinal malasorption syndroms should not use chitosan. Its adverse effects on the growth of children and on the outcome of pregnancy was also reported. However, application of chitin and chitosan have faced some limitation with high viscosity and low solubility at neutral pH (Kim \& Dewapriya 2014).

\section{Conclusion}

Some of the prospects of chitosan in biomedical field have been highlighted and looking potential direction for future research. Furthermore, chitin and chitosan possess various natural biological activities and have a considerable potential to be utilized in a number of medical and industrial applications.

\section{Acknowledgements}

We would like to acknowledge Nepal Academy of Science and Technology (NAST), Lalitpur, Nepal for providing $\mathrm{Ph}$. D. fellowship grant to S. Pokhrel.

\section{References}

Acosta, N., C. Jimenez., V. Borau and A. Heras. 1995. Extraction and characterization of chitin from crustaceans. Biomass Bioenergy 5: 145-153.

Andrady, A.L. and P. Xu. 1997. Elastic behaviour of chitosan films. Journal of Polymer Science Part B: Polymer Physics 35: 517-521.

Carreno-Gomez, B. and A. Duncan. 1997. Evaluation of the biological properties of soluble chitosan and chitosan microspheres. International Journal of Pharmaceutical 148: $231-240$.

Correlo,V.M., L. Bhattacharya, J.F. Mano, N.M. Neves and R.L. Reis. 2005. Properties of melt processed chitosan and aliphatic polyester blends. Material Science and Engineering 403: 57-68.

Corsi, K., F. Chellat, L. Yahia and J.C. Farnandes. 2003. Mesenchymak sten cells, MG63 and HEK293 transfaction using chitosan-DNA nanoparticles. Biomaterials 24: 1255-1261.

Dutta, P.K., J. Dutta and V.S. Tripathi. 2004. Chitin and chitosan: Chemistry, properties and applications. Journal of Scientific and Industrial Research 63: $20-31$.

Elzein, A.R., F. Dabbarh and C. Chaput. 2002. Injectable self-setting calcium phosphate cement. In: Chitosan in pharmacy and chemistry (Eds. R.A.A. Muzzareli \& C. Muzzareli) ATEC: Grottammare. Italy, Pp.365370 . 
Gavhane, Y.N., A.S. Gaurav and A.V. Yadav. 2013. Chitosan and its application: A review of literature. International Journal of Research in Pharmaceutical and Biomedical 4: 312-331.

Han, J.H. 2000. Antimicrobial food packaging. Food Technology 54: 56-65.

Hang, T.T., D.E. Dunstan and D.R. Crispin. 2010. Anticancer activity and therapeutic applications of chitosan nanoparticles. In: Chitin, chitosan, oligosaccharides and their derivatives, biological activities and applications. (Eds. S.K. Kim), New York, CRC Press. UK, Pp. 271-282.

Hench, L.L. 1998. Biomaterial: A forecast for the future. Biomaterials 19: 1419-1423.

Hon, D.N.S. 1996. Chitin and chitosan: Medical applications. In: Polysaccharides in medicinal application (Eds. S. Dumitriu). New York: Marcel Dekker. pp. 631-651.

Hudson, S.M. and C. Smith. 1998. Polysaccharide: Chitin and chitosan: Chemistry and technology of their use as structrual materials. In: Biopolymers from Renewable Resources (Eds. D. L. Kaplan). Pp.96118.

Jianglian, D. and Z. Shaoying. 2013. Application of chitosan based coating in fruits and vegetables preservation: A review. Food Processing and Technology 4: 227-230. doi:10.472/2157-7110.1000227

Jayakumar, R., K.P. Chennazhi, S.V. Nair, T. Furuike and H. Tamura. 2010. Chitosan-conjugated DNA nanoparticle delivery systems for gene therpy. In: Chitin, chitosan, oligosaccharides and their derivatives, biological activities and application (Eds. S.K. Kim) New York, CRC Press. UK, pp.357-366.

Kim, S.K. and P. Dewapriya. 2014. Biologically active compounds from seafood processing by-products. In: Biotransformation of waste biomass into high value biochemicals (Eds. S.K. Brar, G.S. Dhillon \& C.R. Soccol). New York, Heidelberg Dordrecht London. Pp. 299-313.

Khor, E. and L.Y. Lim. 2003. Applications of chitin and chitosan. Biomaterials. 24: 2339-2349.

Majeti, N.V.R. 2000. A review of chitin and chitosan applications. Reactive and Functional Polymer 46: 1-27.

Murakami K., H. Aoki, S. Nakamura, S. Nakamura, M. Takikawa, M. Hanzawa, S. Kishimoto, H. Hattori, Y. Tanaka, T. Kiyosawa, Y. Sato and M. Ishihara. 2010. Hydrogel blends of chitin/chitosan, fucoidan and alginate as healing-impaired wound dressings. Biomaterials 31: 83-90.

Muzzarelli, R.A.A. 1977. Chitin. Pergamon Press Ltd. Headington Hill Hall, Oxford, England Pp.5-37.
Nunthanid, J., M. Luangtana-anam, P. Sriamornsak, S. Limmatvapirat, K. Huanbutta and S. Puttipipatkhachorn. 2009. Use of spray-dried chitosan acetate and ethyl cellulose as compression coats for colonic drug delivery: Effect of swelling on triggering in vitro drug releases. European Journal of Pharmaceutical Biopherma 71: 356-61.

Rinaudo, M. 2006. Chitin and chitosan: Properties and applications. Progress in Polymer Science 31: 603632. doi: 10.1016/J.progpolymsci. 2006.

Saha, S and P. Sarkar. 2013. Removal of arsenic from drinking water using chitosan based mesoporous magnesia impregnated porous alumian: performance evaluation by packed column. International Journal of Environmental Engineering and Management 4(5): 497-498.

Surini, S., H. Akiyama, M. Morishita, T. Nagai and K. Takayama. 2003. Release phenomenon of insulin from an implantable device composed of a ployion complex of chitosan and sodium hyaluronate. Journal of Controlled Release 90: 291-301.

Sridhari, T.R. and P.K. Dutta. 2000. Synthesis and characterization of maleilated chitosan for dye house effluent. Indian Journal of Chemical Technology 7: 198-201.

Vankatesam, J. and S.K. Kim. 2010. Chitosan composites for bone tissue engineering - an overview. Marine Drugs 8: 2252-2266.

Venter, J.P., A.F. Kotze, R. Auzely-Velty and M. Rinauda. 2006. Synthesis and evaluation of mucoadhesivity of CD-chitosan derivative. International Journal of Pharmaceutical 313: 36-42.

Weltrowski, M., B. Martel and M. Morcellet. 1996. Chitosan N-benzyl sulphonate derivatives as sorbents for removal of metal ions in an acidic medium. Journal of Applied Polymer Science 59: 647-654.

Younes, I and M. Rinaudo. 2015. Chitin and chitosan preparation from marine sources structure, properties and applications. Marine Drugs 13: 1133-1174. doi: 103390/md13031133.

Zhang, Y and M. Zhang. 2001. Synthesis and characterization of macroporous chitosan/calcium phosphate composite scaffolds for tissue engineering. Journal of Biomedical Materials Research 55: 304312.

Zhou, Y-G., Y-D. Yang., X-M., X-M. Guo. And G-R. Chen. 2003. Effect of molecular weight and degree of deacetylation of chitosan on urea adsorption properties of copper chitosan. Journal of Applied Polymer Science 89: $1520-1523$.

Zvezdova, D. 2010. Synthesis and characterization of chitosan from marine sources in black sea. Scientific Works of the Rousse University by Bulgaria 49: 65-69. 
Nepal Journal of Science and Technology Vol. 16, No.1 (2015) 99-104 